\title{
The Challenges of Diagnosis in Alzheimer's Disease
}

\author{
An Expert Interview with Nenad Bogdanovic
}

Karolinska University Hospital and Department of Neurobiology, Care Sciences and Society (NVS), Division of Clinical Geriatrics, Karolinska Institutet, Stockholm, Sweden and Geriatric Department, Institute for Clinical Medicine, Oslo University, Oslo, Norway

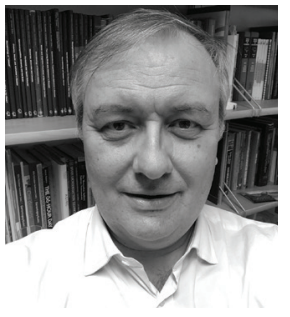

\section{Nenad Bogdanovic}

Nenad Bogdanovic MD PhD, received his medical degree at the University of Zagreb, Croatia, and his PhD degree at the Karolinska Institutet, Stockholm, Sweden. He trained in geriatrics, neurology, neuropathology, and anatomy at the University of Zagreb and then Karolinska University Hospital, where he served most of his career. Previously, he was a Senior Medical Director and Senior Medical Manager for Alzheimer's disease at Wyeth (2007-10) and Pfizer (2010-12), respectively. Dr. Bogdanovic is Professor of Geriatric Medicine at the Karolinska Institutet and Affiliate Professor at Oslo University, Norway. He was a visiting professor at Neural System Lab, Boston University, US in 2013. Currently Dr. Bogdanovic is Head of Neurogeriatric Clinic "The Aging Brain", and Senior Neurogeriatrician at Karolinska University Hospital. He is one of the founders of European BrainNet.

\section{Keywords}

Alzheimer's disease, amyloid-beta, biomarkers, cerebrospinal fluid (CSF), diagnosis, positron emission tomography (PET), tau

Disclosure: Nenad Bogdanovic has nothing to declare in relation to this article.

Review Process: This is an expert interview and as such, has not undergone the journal's standard peer review process.

Authorship: The named author meets the Internationa Committee of Medical Journal Editors (ICMJE) criteria for authorship of this manuscript, takes responsibility for the integrity of the work as a whole, and has given final approval for the version to be published.

Open Access: This article is published under the Creative Commons Attribution Noncommercial License, which permits any noncommercial use, distribution, adaptation, and reproduction provided the original author and source are given appropriate credit. (c) The Author 2018.

Received: January 19, 2018

Published Online: March 14, 2018

Citation: US Neurology. 2018;14(1):15-16

Corresponding Author: Nenad Bogdanovic,

Neurogeriatric Clinic, The Aging Brain, Theme Aging, New Karolinska University Hospital, Huddinge R94, 14186 Stockholm, Sweden. E: Nenad.Bogdanovic@ki.se or Nenad.bogdanovic@karolinska.se

LinkedIn: linkedin.com/in/nenad-bogdanovic-68792916

Support: No funding was received in the publication of this article.
C urrent neuropathologic examination of the brain is still the gold standard for diagnosis of Alzheimer's disease (AD). Postmortem studies, however, have indicated that current methods for the clinical diagnosis of AD are suboptimal. ${ }^{1}$ Recent research has demonstrated the clinical utility of amyloid-beta positron emission tomography (PET) scans, which detect the presence of amyloid-beta plaques in the brain. In a study presented at the Alzheimer's Association International Conference (AAIC) in London, UK, July 2017, by Nenad Bogdanovic, MD, PhD, of the University of Oslo in Norway, amyloid PET imaging was found to be a fundamental diagnostic tool for AD, establishing a definite diagnosis or excluding AD in all 50 study participants. ${ }^{2}$ The use of cerebrospinal fluid (CSF) amyloid testing with a higher amyloid-beta plaque threshold than that traditionally used to establish a positive finding also resulted in high diagnostic accuracy, resulting in diagnosis or exclusion in 44 of 50 participants (88\%) compared with only 21 individuals (42\%) using traditional cutoffs. ${ }^{2}$

In an expert interview following AAIC 2017, Dr. Bogdanovic discusses the challenges of diagnosing AD and future developments in this active area of research.

\section{Q. Why is the diagnosis of Alzheimer's disease (AD) so challenging?}

It is relatively easy to make a diagnosis of dementia, but to determine AD at an early stage is still a clinical challenge. AD is considered as a patho-clinical continuum where neuropathological changes start to accumulate in the brain probably 10-15 years before the first sign of clinical impairment. Dementia due to $A D$ is, in fact, the last stage of the disease. The ability to diagnose AD at an earlier stage would enable intervention with drugs that can specifically target the pathological processes underlying the disease, thus modifying or even halting disease progression. This presents an obvious challenge, as the patient may show no clinical signs of dementia, but subjective cognitive impairment or seemingly unrelated cognitive changes, alterations in personality or mood such as depressive state, neuroticism or changes in social behavior, all of which may have other causes. There is, therefore, a need for biomarkers related to the pathological changes, as well as new imaging approaches that can detect early stages of AD. Understanding the patient's disease stage is very important for prognosis, treatment and appropriate recruitment into clinical trials. Some recent clinical trials may have failed because the patients had an advanced form of the disease or were misdiagnosed (lacking amyloid as a target) and therefore promising antibodies were likely to be "ineffective". ${ }^{3-5}$

\section{Q. What are the limitations of current diagnostic methods for AD?}

The classical approaches to AD diagnosis are thorough neuropsychological evaluation, patient interview, blood-sample analysis and imaging to exclude other, reversible forms of cognitive 
impairment. The use of CSF amyloid and tau analysis is a useful method because it provides information on specific protein changes in the brain, and is frequently used in Scandinavian countries. However, there is high inter- and intra-laboratory variation so the classical cutoff of pathological levels varies between centers performing the same methods. The major limitation of current approaches is that there are no useful markers of disease stage. Markers have been developed for brain atrophy, but the latter is not always present at early diagnosis. Other advanced methods like PET-amyloid camera are not available in every clinical setting, which limits the accuracy of diagnosis. Outside of universities and neurological clinics, diagnostic methods are simpler and even limited, thus misdiagnoses are more frequent. The testing of other cognitive domains besides memory impairment is still not standardized for AD. Furthermore, the knowledge of neuropathological processes in $A D$ and normal aging are not entirely understood, especially in an elderly population where a similar clinical picture might have a different neurobiological background. We still have lot to learn from neuropathology. Earlier diagnosis is imperative, as it may enhance the ability to study disease course and clinical heterogeneity, predict the future and personalize treatment. We need a consensus approach.

\section{Q. What methods are currently under investigation?}

Methods specifically directed to AD biomarkers such as PET-amyloid, CSF amyloid-beta and tau are widely accepted by regulatory authorities. PET-tau is under intense development, and many studies have identified potential imaging ligands for tau, but no standardized methods are yet available. New technology in CSF analysis aims to decrease the variability in CSF amyloid testing from $15 \%$ to around $2 \%$, making it easier to define cutoffs for patients with mild cognitive impairment and with AD. Besides standard biomarkers of amyloid and tau, CSF is a medium where synaptic proteins and neurofilament are currently under intensive and promising clinical research and early applications. There is great interest in identifying standard AD biomarkers such as amyloid and tau in the blood because of relative ease of access and patient comfort. The important question is whether presymptomatic AD causes consistent, measurable changes in urine or blood levels of tau, beta-amyloid or other biomarkers. Moreover, of interest is to investigate whether early AD leads to detectable biomarker proteins changes elsewhere in the body, such as deposits in the lens of the eye. Combined approaches involving genetics and expression of different pathological proteins can also give some idea of the pathophysiological pathways and disease stage.

\section{Q. Among these, which do you consider most promising and why?}

Tau imaging is the most promising method currently under investigation and is close to gaining acceptance for routine clinical use. Once a PET-tau ligand is accepted by regulatory authorities we would be able to visualize the two most important AD pathological hallmarks, which will allow not only a very precise analysis of disease staging, but will also help clinicians in differential diagnoses against other dementia disorders. Blood biomarkers are also a highly promising approach but still require further development.

\section{Q. What other blood-based biomarkers are being evaluated for $A D$ ?}

The challenge with blood-based biomarkers is failure to replicate findings preventing widespread acceptance of any blood-based assay for AD. Blood is very complex, characterized by multiple cellular compartments and an ever-changing environment of proteins, lipids, and other biochemical entities that affect standardization and reproducibility of results. Specifically, participant's age, cognitive health of subjects, diet of the participants, the assay used, inter-lab variability and little correspondence with clinical stage are some of the major obstacles that should be resolved. Recently some other $A \beta$ species, particularly $A \beta_{1-17}$, have been proposed to be useful for the diagnosis of AD. ${ }^{6}$ Neurofilament light chain protein also appears to be a promising marker of disease in preclinical studies of $A D,{ }^{7}$ and its elevation in CSF may help in making differential diagnoses of some genetic forms of frontal lobe dementia..$^{8} \square$

\footnotetext{
Beach TG, Monsell SE, Phillips LE, Kukull W. Accuracy of the clinical diagnosis of Alzheimer disease at National Institute on Aging Alzheimer Disease Centers, 2005-2010. J Neuropathol Exp Neurol. 2012;71:266-73. Bogdanovic N, Farrar G, Fantoni ER. Measurement of pathological amyloid in routine clinical assessment: the clinical impact of visual [18F]flutemetamol PET and CSF analysis. Alzheimers Dement. 2017;13 Suppl. P411. Karran E, Hardy J. Antiamyloid therapy for Alzheimer's disease - are we on the right road? N Engl J Med. 2014;370:377-8.

Doody RS, Thomas RG, Farlow M, et al. Phase 3 trials of solanezumab for mild-to-moderate Alzheimer's disease. N Eng/ J Med. 2014;370:311-21.

Salloway S, Sperling R, Fox NC, et al. Two phase 3 trials of bapineuzumab in mild-to-moderate Alzheimer's disease. N Engl J Med. 2014;370:322-33.

Pérez-Grijalba V, Pesini P, Allué JA, et al. A 1 1-17 is a major amyloid- $\beta$ fragment isoform in cerebrospinal fluid and blood with possible diagnostic value in Alzheimer's disease. J Alzheimers Dis. $2015 ; 43: 47-56$.

Mattsson N, Andreasson U, Zetterberg H, et al. Association of plasma neurofilament light with neurodegeneration in patients with Alzheimer disease. JAMA Neurol. 2017;74:557-66.

Meeter LH, Dopper EG, Jiskoot LC, et al. Neurofilament light chain: a biomarker for genetic frontotemporal dementia. Ann Clin Trans/ Neurol. 2016;3:623-36.
} 